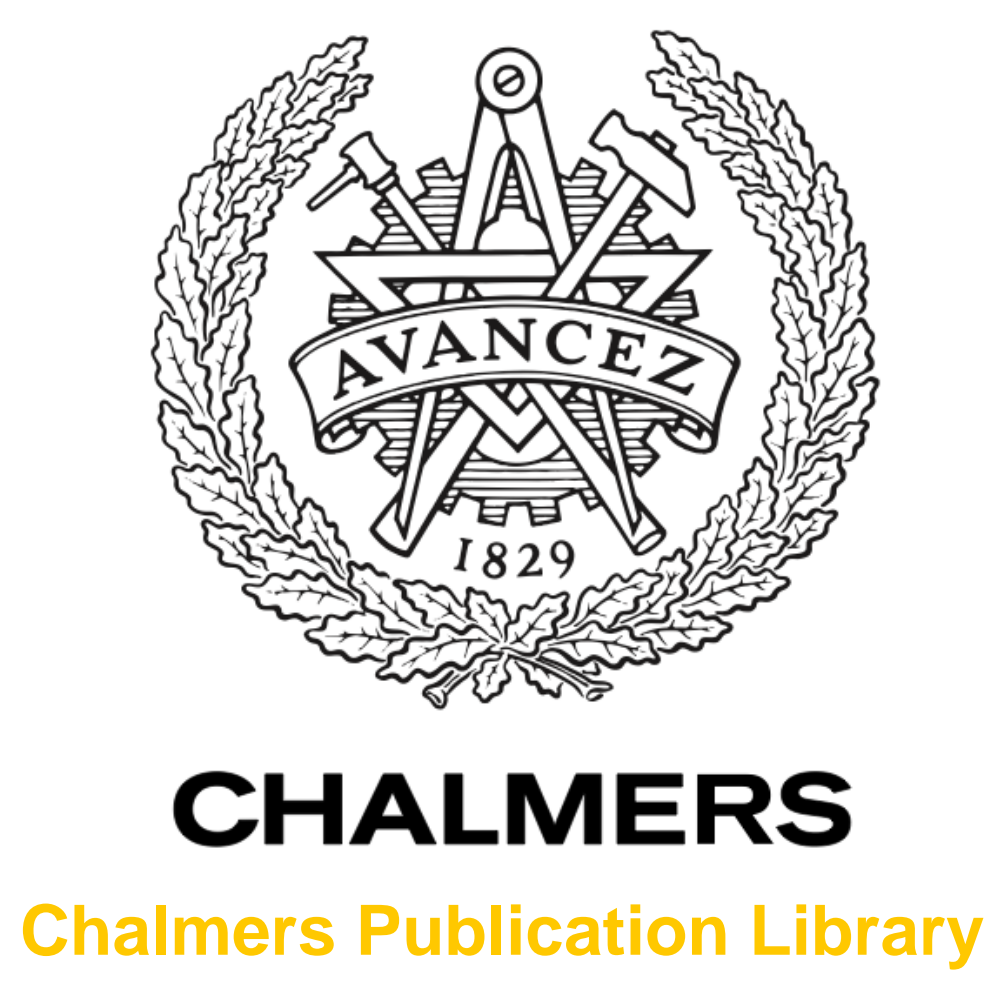

\title{
Wave propagation in 2D elastic composites with partially debonded fibres by the null field appraoch
}

This document has been downloaded from Chalmers Publication Library (CPL). It is the author's version of a work that was accepted for publication in:

Waves in Random and Complex Media (ISSN: 1745-5030)

Citation for the published paper:

Matus, V. ; Kunets, Y.; Mykhavskiv, V. et al. (2009) "Wave propagation in 2D elastic composites with partially debonded fibres by the null field appraoch". Waves in Random and Complex Media, vol. 19(4), pp. 654-669.

http://dx.doi.org/10.1080/17455030903267087

Downloaded from: http://publications.lib.chalmers.se/publication/103484

Notice: Changes introduced as a result of publishing processes such as copy-editing and formatting may not be reflected in this document. For a definitive version of this work, please refer to the published source. Please note that access to the published version might require a subscription.

Chalmers Publication Library (CPL) offers the possibility of retrieving research publications produced at Chalmers University of Technology. It covers all types of publications: articles, dissertations, licentiate theses, masters theses, conference papers, reports etc. Since 2006 it is the official tool for Chalmers official publication statistics. To ensure that Chalmers research results are disseminated as widely as possible, an Open Access Policy has been adopted.

The CPL service is administrated and maintained by Chalmers Library. 


\title{
Wave propagation in 2-D elastic composites with partially debonded fibers by the null field approach
}

\author{
V. Matus a, Y. Kunets ${ }^{\mathrm{a}}$, V. Mykhas'kiva,*, \\ A. Boström ${ }^{\mathrm{b}}$, Ch. Zhang ${ }^{\mathrm{c}}$ \\ ${ }^{a}$ Pidstryhach Institute for Applied Problems of Mechanics and Mathematics, Lviv 79060, Uk raine; ${ }^{\mathrm{b}}$ Department of Applied \\ Mechanics, Chalmers University of Technology, Goteborg 41296, Sweden; ${ }^{\mathrm{c}}$ Department of Civil Engineering, University of \\ Siegen, Siegen 57068, Germany

\begin{abstract}
Time-harmonic plane wave propagation in a two-dimensional (2-D) elastic matrix with partially debonded elastic fibers of nonclassical cross-section is investigated. The modified null field approach, taking into account the asymptotic behavior of the solution at the interface crack-tips, is exploited to obtain the numerical results for a single scatterer. The effective medium approach based on Foldy's approximation is applied to estimate the average dynamic parameters of the composites containing randomly distributed partially debonded fibers of dilute distributed partially debonded fibers of dilute concentration. Numerical results concen the longitudinal wave dispersion and crack) size and direction of wave incidence on the effective P-wave velocity and attenuation coefficient are analyzed.
\end{abstract} \\ Keywords: elastodynamic wave scattering; 2-D elastic composites; inclusion of non-classical shape; interface crack; effective
} dynamic properties; modified null field approach.

\section{Introduction}

Fiber-reinforced composites are used widely as structural components in many engineering applications. In many cases the degradation of physical properties of such composites is caused by the debonding of fibers. This fact should be accounted for when studying the elastic wave propagation in fiber-reinforced composites, for example, for their adequate ultrasonic non-destructive evaluation (see Achenbach [1]). Additional interest lies in considering non-circular fibers, which can give rise to some special properties of composite materials in the average sense, as was shown by Bond et al. [2].

The effective dynamic characteristics of fiber-reinforced composites with partially debonded elastic fibers have been investigated by several authors. Phase velocity and attenuation of an SH-wave in a composite with circular fibers were calculated by Zhang and Gross [3]. Sato and Shindo [4] examined the effective characteristics of $\mathrm{P}$ - and SV-waves in elastic composites with elliptical inclusions. The solutions of elastodynamic problems for a single scatterer are a necessary constructive part of such investigations. In this aspect Yang and Norris [5, 6], Zhang and Gross [3], and Wang and Wang [7] discussed the scattering of elastic waves by partially debonded circular elastic or rigid inclusions. Sato and Shindo [4] considered such a problem for an elongated elliptical elastic inclusion with an interface crack. Diffraction of an SH-wave by a debonded elastic inclusion of more complicated square and triangular shapes was studied by Kunets et al. [8]. However, the extension of

\footnotetext{
* Corresponding author. Tel.: +380 322399911

E-mail address: tex@iapmm.lviv.ua (V.V. Mykhas’kiv)
} 
these methods to the analysis of the macroscopic dynamic behavior of composites with distributed debonded inclusions of general shapes was not realized until now.

In this paper we analyze the effective dynamic characteristics of two-dimensional (2-D) elastic composites with partially debonded elastic inclusions by using the null field approach. Previously this method has been successfully applied for the time-harmonic analysis of electromagnetic and acoustic scattering by Waterman [9]. Many references on the subject can be found in the papers by Varatharajulu and Pao [10], Olsson and Boström [11], Boström et al. [12], Emets et al. [13] and in the review works by Martin [14], Beskos [15], and Boström [16]. Boström and Olsson [17] proposed a modification of the null field approach to study the scattering of elastic waves by non-planar cracks, where the "square-root" behavior of the solution at the crack-tip is explicitly considered. In the present paper this approach is implemented for an interface crack, when the unknown displacements and stresses at the interface have an oscillatory character in a small region around the crack-tips. Such an approach may considerably increase the accuracy in the calculation of the scattering parameters. By using the null field approach and Foldy's relations [18], the effects of the fiber shape, debonding (interface crack) size and direction of wave incidence on the effective P-wave velocity and attenuation coefficient are analyzed.

\section{Wave scattering by a single inclusion and the null field approach}

Consider an unbounded elastic matrix $W_{1}$ with the mass density $\rho_{1}$, Lamé parameters $\lambda_{1}$ and $\mu_{1}$ containing an elastic inclusion with corresponding material parameters $\rho_{2}, \lambda_{2}$ and $\mu_{2}$, which occupies the region $W_{2}$. An interface crack is located on a part $S_{0}$ of the matrix-fiber interface $S$ and the conditions of a perfect (or ideal) contact are assumed on the part $S_{1}=S \backslash S_{0}$ (see Figure 1). The elastic composite is in time-harmonic motion, therefore the common factor exp $(-i \omega t)$ can be omitted, where $i$ is the imaginary unit, $\omega$ is the circular frequency and $t$ is the time. The longitudinal and

transverse wavenumbers in the matrix are $k_{p 1}=\omega\left[\rho_{1} /\left(\lambda_{1}+2 \mu_{1}\right)\right]^{1 / 2}$ and $k_{s 1}=\omega\left(\rho_{1} / \mu_{1}\right)^{1 / 2}$, respectively, and similarly for $k_{p 2}$ and $k_{s 2}$ in the inclusion. The displacement vectors for the plane strain problem both in the matrix and the inclusion $\boldsymbol{u}^{1}(\boldsymbol{x})$ and $\boldsymbol{u}^{2}(\boldsymbol{x})$, respectively, satisfy the equations of motion [19]

$$
\left(\lambda_{j}+\mu_{j}\right) \operatorname{grad} \operatorname{div} \boldsymbol{u}^{j}(\boldsymbol{x})+\mu_{j} \nabla^{2} \boldsymbol{u}^{j}(\boldsymbol{x})+\mu_{j} k_{s j}^{2} \boldsymbol{u}^{j}(\boldsymbol{x})=0, \quad \boldsymbol{x} \in W_{j}, \quad j=1,2,
$$

and the following boundary and continuity conditions 


$$
\begin{gathered}
\boldsymbol{u}^{1}=\boldsymbol{u}^{2}, \quad \boldsymbol{t}^{1}\left(\boldsymbol{u}^{1}\right)=\boldsymbol{t}^{2}\left(\boldsymbol{u}^{2}\right), \quad \boldsymbol{x} \in S_{1}, \\
\boldsymbol{t}^{1}\left(\boldsymbol{u}^{1}\right)=0, \quad \boldsymbol{t}^{2}\left(\boldsymbol{u}^{2}\right)=0, \quad \boldsymbol{x} \in S_{0}, \\
\boldsymbol{t}^{j}\left(\boldsymbol{u}^{j}\right)=n \lambda_{j} \nabla \cdot \boldsymbol{u}^{j}+2 \mu_{j} \frac{\partial \boldsymbol{u}^{j}}{\partial n}+\mu_{j} \boldsymbol{n} \times\left(\nabla \times \boldsymbol{u}^{j}\right) .
\end{gathered}
$$

Here $\boldsymbol{x}=\left(x_{1}, x_{2}\right)$ is the position vector, $\boldsymbol{t}^{j}\left(\boldsymbol{u}^{j}\right)$ is the traction vector and $\boldsymbol{n}$ is the outer unit normal vector to $S$.

The scattered field $\boldsymbol{u}^{s}(\boldsymbol{x})$ is introduced as the difference between the total field $\boldsymbol{u}(\boldsymbol{x})$ and the incident one $\boldsymbol{u}^{\text {in }}(\boldsymbol{x})$ in $W_{1}$, namely

$$
\boldsymbol{u}^{s}(\boldsymbol{x})=\boldsymbol{u}^{1}(\boldsymbol{x})-\boldsymbol{u}^{i n}(\boldsymbol{x})
$$

To completely formulate the scattering problem the radiation conditions at infinity are required. The scattered displacements in the far-field can be written as

$$
\boldsymbol{u}^{s}(\boldsymbol{x})=\sum_{\alpha=p, s} \frac{\exp \left(i k_{\alpha 1} r+i \pi / 4\right)}{\left(8 \pi k_{\alpha 1} r\right)^{1 / 2}} \boldsymbol{f}^{\alpha}(\theta)+O\left(r^{-1}\right), \quad r \rightarrow \infty, \quad \boldsymbol{x}=(r \cos \theta, r \sin \theta) .
$$

Here $f^{\alpha}(\theta)$ is the far-field amplitude vector of the scattered longitudinal $(\alpha=p)$ and transverse $(\alpha=s)$ waves and $(r, \theta)$ are polar coordinates.

The incident and scattered wave fields are expanded into the cylindrical waves by the formula [10]

$$
\begin{gathered}
\boldsymbol{u}^{i n}(\boldsymbol{x})=u_{0} \sum_{\alpha, \sigma, m} a_{\alpha \sigma m}^{i n} \operatorname{Re} \boldsymbol{\Phi}_{\alpha \sigma m}^{1}(\mathbf{x}), \quad \boldsymbol{u}^{s}(\boldsymbol{x})=u_{0} \sum_{\alpha, \sigma, m} f_{\alpha \sigma m} \boldsymbol{\Phi}_{\alpha \sigma m}^{1}(\boldsymbol{x}), \\
\boldsymbol{\Phi}_{p \sigma m}^{j}(\boldsymbol{x})=\varepsilon_{m}^{1 / 2} \nabla \psi_{p \sigma m}^{j}(\boldsymbol{x}), \quad \boldsymbol{\Phi}_{s \sigma m}^{j}(\boldsymbol{x})=\varepsilon_{m}^{1 / 2} \nabla \times \boldsymbol{e}_{z} \psi_{s \sigma m}^{j}(\boldsymbol{x}), \quad j=1,2, \\
\psi_{\alpha \sigma m}^{j}(\boldsymbol{x})=H_{m}^{(1)}\left(k_{\alpha j} r\right) C_{\sigma m}(\theta), \quad C_{\sigma m}(\theta)=\left\{\begin{array}{l}
\cos m \theta, \sigma=1, m=0,1, \ldots, \\
\sin m \theta, \sigma=2, m=1,2, \ldots,
\end{array}\right.
\end{gathered}
$$

where $u_{0}$ is the amplitude of the incident wave, $\left(\boldsymbol{e}_{r}, \boldsymbol{e}_{\theta}, \boldsymbol{e}_{z}\right)$ are the unit vectors in cylindrical coordinates, $H_{m}^{(1)}(r)$ is the Hankel function of the $m$-th order and the first kind [20], $\varepsilon_{m}=2-\delta_{m 0}$, $\delta_{m 0}$ is the Kronecker delta and Re stands for the real or regular part, i.e. Bessel instead of Hankel functions. Here, the coefficients $a_{\alpha \sigma m}^{i n}$ are known for a fixed angle $\theta_{i n}$ of the wave incidence and are 
linearly related to the unknown coefficients $f_{\alpha \sigma m}$ by the transition matrix (T-matrix) $T_{\alpha \sigma m, \alpha^{\prime} \sigma^{\prime} m^{\prime}}$ [17] as following

$$
f_{\alpha \sigma m}=\sum_{\alpha^{\prime}, \sigma^{\prime}, m^{\prime}} T_{\alpha \sigma m, \alpha^{\prime} \sigma^{\prime} m^{\prime}} a_{\alpha^{\prime} \sigma^{\prime} m^{\prime}}^{i n}
$$

In particular, for an incident plane longitudinal P-wave we have

$$
\boldsymbol{u}^{i n}(\boldsymbol{x})=u_{0} \boldsymbol{g} \exp \left(i k_{p 1} \boldsymbol{g} \cdot \boldsymbol{x}\right), \quad a_{p \sigma m}^{i n}=i^{-m-1} \varepsilon_{m}^{1 / 2} C_{\sigma m}\left(\theta_{i n}\right) / k_{p 1}, \quad a_{s \sigma m}^{i n}=0,
$$

and for an incident plane transverse S-wave we have

$$
\boldsymbol{u}^{i n}(\boldsymbol{x})=-u_{0} \boldsymbol{g} \times \boldsymbol{e}_{z} \exp \left(i k_{s 1} \boldsymbol{g} \cdot \boldsymbol{x}\right), \quad a_{p \sigma m}^{i n}=0, \quad a_{s \sigma m}^{i n}=i^{-m+1} \varepsilon_{m}^{1 / 2} C_{\sigma m}\left(\theta_{i n}\right) / k_{s 1},
$$

where the unit vector $g=-\left(\cos \theta_{i n}, \sin \theta_{i n}\right)$ defines the incidence direction of the plane wave.

With the purpose of finding a numerical solution of the exterior problem (1) - (4) in the far-field of a scatterer the null field approach is used. Then, from the outer and the inner integral representations we can obtain

$$
\begin{gathered}
\int_{S}\left[\boldsymbol{u}^{1} \cdot \boldsymbol{t}^{1}\left(\boldsymbol{\Phi}_{\alpha \sigma m}^{1}\right)-\boldsymbol{\Phi}_{\alpha \sigma m}^{1} \cdot \boldsymbol{t}^{1}\left(\boldsymbol{u}^{1}\right)\right] d S=4 i u_{0} \mu_{1} k_{s 1}^{2} a_{\alpha \sigma m}^{i n}, \\
\int_{S}\left[\boldsymbol{u}^{2} \cdot \boldsymbol{t}^{2}\left(\operatorname{Re} \boldsymbol{\Phi}_{\alpha \sigma m}^{2}\right)-\boldsymbol{t}^{2}\left(\boldsymbol{u}^{2}\right) \cdot \operatorname{Re} \boldsymbol{\Phi}_{\alpha \sigma m}^{2}\right] d S=0 .
\end{gathered}
$$

The representation of the unknown functions on $S$ can be realized in different ways. Here, we use the modified null field approach developed by Boström and Olsson [17], in which the behavior of the solution at the crack-tips is accounted for explicitly. The asymptotic behavior of the displacements and stresses at the crack-tips can be determined by the technique proposed by Dempsey and Sinclair [21] or Kondrat'ev and Oleinik [22]. Accordingly we have the following asymptotic approximations

$$
\begin{gathered}
\boldsymbol{u}^{1}-\boldsymbol{u}^{2} \approx R_{0}^{1 / 2}\left[R_{0}^{i \delta}(\boldsymbol{n}-i \tau) D_{1}+R_{0}^{-i \delta}(\boldsymbol{n}+i \tau) D_{2}\right], \quad \text { on } S_{0}, \\
\boldsymbol{t}^{2} \approx \frac{\mu_{2} G}{a R_{0}^{1 / 2}}\left[R_{0}^{i \delta}(1+2 i \delta)(\boldsymbol{n}-i \tau) D_{1}+R_{0}^{-i \delta}(1-2 i \delta)(\boldsymbol{n}+i \tau) D_{2}\right], \quad \text { on } S_{1}, \quad R_{0} \rightarrow 0, \\
\delta=\frac{1}{2 \pi} \ln \frac{\mu_{1}+\mu_{2}\left(3-4 v_{2}\right)}{\mu_{2}+\mu_{1}\left(3-4 v_{1}\right)}, \quad G=\mu_{1}\left(\mu_{1}+\mu_{2}\left(3-4 v_{1}\right)\right)^{-1 / 2}\left(\mu_{2}+\mu_{1}\left(3-4 v_{2}\right)\right)^{-1 / 2},
\end{gathered}
$$

where $R_{0}$ is the, by the characteristic dimension $a$ of the inclusion, normalized distance to the cracktip, $D_{1}$ and $D_{2}$ are arbitrary amplitudes in the asymptotic approximations, $v_{1}$ and $v_{2}$ are Poisson's 
ratios of the matrix and the inclusion, respectively, and $\boldsymbol{n}$ and $\boldsymbol{\tau}$ are the unit normal and tangential vectors to $S$ (Figure 1).

Similarly as in [17], introduce the auxiliary field

$$
\boldsymbol{v}(\theta)= \begin{cases}\mathbf{Z}^{-1} \cdot\left(\boldsymbol{u}^{1}-\boldsymbol{u}^{2}\right), & \text { on } S_{0}, \\ \frac{a}{\mu_{2}} \mathbf{Z}^{-1} \cdot \boldsymbol{t}^{2}, & \text { on } S_{1},\end{cases}
$$

where

$$
\begin{gathered}
\mathbf{Z}= \begin{cases}R^{1 / 2}\left(\mathbf{Z}_{1}+\mathbf{Z}_{2}\right), & \text { on } S_{0}, \\
G R^{-1 / 2}\left[(1+2 i \delta) \mathbf{Z}_{1}+(1-2 i \delta) \mathbf{Z}_{2}\right], & \text { on } S_{1},\end{cases} \\
\mathbf{Z}_{1}=R^{i \delta}(\boldsymbol{n n}-i \tau \boldsymbol{n}), \quad \mathbf{Z}_{2}=R^{-i \delta}(\boldsymbol{n} \tau+i \tau \tau), \quad R=a^{-1}\left|\boldsymbol{x}-\boldsymbol{x}_{0}\right|\left|\boldsymbol{x}-\boldsymbol{x}_{1}\right|\left|\boldsymbol{x}_{1}-\boldsymbol{x}_{0}\right|^{-1},
\end{gathered}
$$

in which $\boldsymbol{x}_{0}$ and $\boldsymbol{x}_{1}$ are position vectors of the crack-tips with the angle coordinates $\theta_{0}$ and $\theta_{1}$ (Figure 1). The dyadic $\mathbf{Z}$ is introduced so that the crack-tip conditions (9) are considered explicitly. With this choice of $\mathbf{Z}$ the surface field $\boldsymbol{v}$ as defined in (10) will be both finite and continuous in the vicinity of the crack-tip. The unknown stresses and displacements in (8) can be expressed in terms of the functions $\boldsymbol{v}(\theta)$ and $\boldsymbol{u}^{2}(\theta)$, which are thus continuous on $S$. Therefore, it is convenient to expand these functions into the following series

$$
\begin{gathered}
\boldsymbol{v}(\theta)=u_{0} \sum_{\sigma, m} \boldsymbol{y}_{1 \sigma m} C_{\sigma m}(\theta), \quad \boldsymbol{u}^{2}(\theta)=u_{0} \sum_{\sigma, m} \boldsymbol{y}_{2 \sigma m} C_{\sigma m}(\theta), \quad \text { on } S, \\
\boldsymbol{y}_{l \sigma m}=y_{l 1 \sigma m} \boldsymbol{n}+y_{l 2 \sigma m} \boldsymbol{\tau}, \quad l=1,2,
\end{gathered}
$$

where $\boldsymbol{y}_{l \sigma m}(l=1,2)$ are the unknown coefficients of the expansions (11).

Substituting the expansions (11) into the equations (8) and using the boundary and continuity conditions (2) and (3) we obtain the following infinite system of linear algebraic equations for the coefficients $\boldsymbol{y}_{l \sigma m}(l=1,2)$

$$
\begin{gathered}
\sum_{\alpha^{\prime}, \sigma^{\prime}, m^{\prime}}\left(a_{\alpha \sigma m, \alpha^{\prime} \sigma^{\prime} m^{\prime}}^{1} y_{1 \alpha^{\prime} \sigma^{\prime} m^{\prime}}+a_{\alpha \sigma m, \alpha^{\prime} \sigma^{\prime} m^{\prime}}^{2} y_{2 \alpha^{\prime} \sigma^{\prime} m^{\prime}}\right)=4 i k_{s 1}^{2} \mu_{1} a_{\alpha \sigma m}^{i n}, \\
\sum_{\alpha^{\prime}, \sigma^{\prime}, m^{\prime}}\left(a_{\alpha \sigma m, \alpha^{\prime} \sigma^{\prime} m^{\prime}}^{3} y_{1 \alpha^{\prime} \sigma^{\prime} m^{\prime}}+a_{\alpha \sigma m, \alpha^{\prime} \sigma^{\prime} m^{\prime}}^{4} y_{2 \alpha^{\prime} \sigma^{\prime} m^{\prime}}\right)=0
\end{gathered}
$$




$$
\begin{gathered}
a_{\alpha \sigma m, \alpha^{\prime} \sigma^{\prime} m^{\prime}}^{1}=\boldsymbol{e}_{\alpha^{\prime}} \cdot\left\{\int_{S_{0}} \mathbf{Z} \cdot \boldsymbol{t}^{1}\left(\boldsymbol{\Phi}_{\alpha \sigma m}^{1}\right) C_{\sigma^{\prime} m^{\prime}}(\theta) d S-\frac{\mu_{2}}{a} \int_{S_{1}} \mathbf{Z} \cdot \boldsymbol{\Phi}_{\alpha \sigma m}^{1} C_{\sigma^{\prime} m^{\prime}}(\theta) d S\right\}, \\
a_{\alpha \sigma m, \alpha^{\prime} \sigma^{\prime} m^{\prime}}^{2}=\boldsymbol{e}_{\alpha^{\prime}} \cdot \int_{S} \boldsymbol{t}^{1}\left(\boldsymbol{\Phi}_{\alpha \sigma m}^{1}\right) C_{\sigma^{\prime} m^{\prime}}(\theta) d S, \\
a_{\alpha \sigma m, \alpha^{\prime} \sigma^{\prime} m^{\prime}}^{3}=-\frac{\mu_{2}}{a} \boldsymbol{e}_{\alpha^{\prime}} \cdot \int_{S} \operatorname{Re}\left(\boldsymbol{\Phi}_{\alpha \sigma m}^{2}\right) C_{\sigma^{\prime} m^{\prime}}(\theta) d S, \\
a_{\alpha \sigma m, \alpha^{\prime} \sigma^{\prime} m^{\prime}}^{4}=\boldsymbol{e}_{\alpha^{\prime}} \cdot \int_{S} \boldsymbol{t}^{2}\left(\operatorname{Re} \boldsymbol{\Phi}_{\alpha \sigma m}^{2}\right) C_{\sigma^{\prime} m^{\prime}}(\theta) d S, \quad \alpha^{\prime}=1,2, \quad \boldsymbol{e}_{1}=\boldsymbol{n}, \quad \boldsymbol{e}_{2}=\boldsymbol{\tau} .
\end{gathered}
$$

In the numerical computations the summation index $m$ has to be truncated at some limit $m_{\max }$. Indeed, in our analysis an acceptable accuracy is obtained if we take $m_{\max }=6+m_{d}$ for $k_{p 1} a \leq 3$ and $m_{\max }=2 k_{p 1} a+m_{d}$ (truncated to closest integer) for $3<k_{p 1} a \leq 5$. The parameter $m_{d}$ is determined by numerical experiments. In principle, there is no problems to go to higher wavenumbers (frequencies), but then the value of the truncation parameter must be rechecked.

As the result of equation (4) and the integral representation for $\boldsymbol{u}^{s}(\boldsymbol{x})$ [10], the far-field amplitude is determined by the formula

$$
\begin{gathered}
\boldsymbol{f}^{p}(\theta)=u_{0} f^{p}(\theta) \boldsymbol{e}_{r}, \quad \boldsymbol{f}^{s}(\theta)=u_{0} f^{s}(\theta) \boldsymbol{e}_{\theta} \\
f^{\alpha}(\theta)=\frac{(-1)^{\delta_{\alpha s}} i k_{\alpha 1}}{u_{0} \mu_{1} k_{s 1}^{2}} \sum_{\sigma, m}\left\{\varepsilon_{m}^{1 / 2} i^{-m} C_{\sigma m}(\theta) \int_{S}\left[\boldsymbol{u}^{1} \cdot \boldsymbol{t}^{1}\left(\operatorname{Re} \boldsymbol{\Phi}_{\alpha \sigma m}^{1}\right)-\boldsymbol{t}^{1}\left(\boldsymbol{u}^{1}\right) \cdot \operatorname{Re} \boldsymbol{\Phi}_{\alpha \sigma m}^{1}\right] d S\right\}
\end{gathered}
$$

Equation (13) can be rewritten in the form

$$
f^{\alpha}(\theta)=4(-1)^{\delta_{\alpha s}} k_{\alpha 1} \sum_{\sigma, m} \sum_{\alpha^{\prime}, \sigma^{\prime}, m^{\prime}} \varepsilon_{m}^{1 / 2} i^{-m} C_{\sigma m}(\theta) T_{\alpha \sigma m, \alpha^{\prime} \sigma^{\prime} m^{\prime}} a_{\alpha^{\prime} \sigma^{\prime} m^{\prime}}^{i n}, \quad \alpha^{\prime}=p, s,
$$

where $T_{\alpha \sigma m, \alpha^{\prime} \sigma^{\prime} m^{\prime}}$ are the elements of the corresponding T-matrix introduced by equation (6). They follow from the inversion of equations (12) and the subsequent substitution of the solution into the representations (11) and (13).

Consequently, the expressions (13) and (14) give the solution of the scattered displacements in the far-field of a scatterer. 


\section{Homogenization for distributed inclusions with interface cracks}

For the sake of brevity, let us consider the situation when a plane longitudinal P-wave propagates in an elastic composite containing multiple debonded inclusions with a uniformly random distribution. Note here that the analysis can be easily extended to the transverse S-wave propagation in a similar manner. The location of the inclusions is assumed to be random, while their orientation is either completely random or aligned. In addition the shapes and the cross-sectional areas of the inclusions as well as the size of their debonding are assumed to be equal. The average response of such a composite solid to the wave propagation is characterized by the wave dispersion and attenuation due to the scattering process. These properties can be described by an effective medium approach with a complex and frequencydependent wave number $K_{p}(\omega)$ represented as

$$
K_{p}(\omega)=\frac{\omega}{c_{p}(\omega)}+i \alpha_{p}(\omega)
$$

where $c_{p}(\omega)$ is the effective P-wave (phase) velocity and $\alpha_{p}(\omega)$ is the attenuation coefficient.

For a low concentration of fibers the multiple scattering effects among individual fibers can be neglected. Thus the effective wavenumber $K_{p}(\omega)$ can be calculated by using Foldy's dispersion relation in the form

$$
K_{p}^{2}=k_{p 1}^{2}+\frac{\varepsilon}{\pi a^{2}} F_{p}
$$

In equation (16), $\varepsilon$ is the inclusion density parameter and the quantity $\varepsilon /\left(\pi a^{2}\right)$ corresponds to the number density of the inclusions, i.e. the number of inclusions per unit area, and $F_{p}$ is the average forward scattering amplitude of P-waves for a single debonded fiber. For the equally-shaped and equally-debonded randomly oriented inclusions the average should be performed only over all possible orientations. Noting that the mean over all orientations is the same as the mean over all directions of the wave incidence, hence

$$
F_{p}=\left\{\begin{array}{l}
\frac{1}{2 \pi} \int_{0}^{2 \pi} f^{p}\left(\pi+\theta_{i n}\right) d \theta_{i n}, \text { for randomly oriented inclusions, } \\
f^{p}\left(\pi+\theta_{i n}\right), \text { for aligned inclusions. }
\end{array}\right.
$$

It should be mentioned that, similarly to the paper by Eriksson et al. [23] for the case of randomly oriented inclusions, the average forward scattering amplitude and the T-matrix elements are related by 


$$
F_{p}=-4 i \sum_{\sigma, m} T_{p \sigma m, p \sigma m}
$$

Once the complex effective wavenumber $K_{p}$ has been determined via equations (16) and (17), the normalized effective wave velocity $\bar{c}=c_{p}(\omega) / c_{p 1}$ and the normalized attenuation coefficient $\bar{\alpha}=2 \pi a \alpha_{p} / \varepsilon$ of the plane P-wave in the composite can be obtained by using equation (15), where $c_{p 1}=\left[\left(\lambda_{1}+2 \mu_{1}\right) / \rho_{1}\right]^{1 / 2}$ is the phase velocity of the longitudinal P-wave in the matrix. That results in

$$
\bar{c}(\omega)=\frac{k_{p 1}}{\operatorname{Re}\left[K_{p}(\omega)\right]}, \quad \bar{\alpha}(\omega)=\frac{2 \pi a}{\varepsilon} \operatorname{Im}\left[K_{p}(\omega)\right] .
$$

\section{Numerical examples}

The method proposed above is used to study the plane time-harmonic longitudinal wave propagation through fibrous composites in the presence of debondings between the matrix and the fibers. The shape of the cross-section of the fibers is given by

$$
\begin{gathered}
r(\beta)=a \sqrt{\frac{1+\gamma^{2}-2 \gamma \cos (N+1) \beta}{1-\gamma^{2} N}}, \\
\theta(\beta)=\arctan \frac{\sin \beta+\gamma \sin N \beta}{\cos \beta-\gamma \cos N \beta}, \quad 0 \leq \beta \leq 2 \pi,
\end{gathered}
$$

where $N=1$ is for an ellipse and $N=3$ for a quasi-square with rounded corners. Here the crosssections of the inclusions have equal areas and are parameterized by fixing $\gamma$, so that the circular inclusion with radius $a$ has $\gamma=0$.

Calculations have been performed for three combinations of matrix and fiber: (i) type I: epoxy/glass composite; (ii) type II: epoxy/glass composite; (iii) type III: $\mathrm{Al} / \mathrm{SiC}$ composite. The corresponding material constants are given in Table 1. The elastic moduli in composite II are selected so that the oscillation coefficient $\delta=0$ in (9). For the numerical solutions the truncation parameter $m_{d}$ was chosen in the range from 3 to 9 depending on the fiber geometry.

To illustrate the accuracy of the proposed method we present in Figure 2 the frequency dependence of the normalized scattering cross section $\sigma=\left(k_{p 1} a\right)^{-1} \operatorname{Im}\left[f^{p}\left(\pi+\theta_{i n}\right)\right]$ for the P-wave scattering by a single circular elastic inclusion with a debonding for the composite II, which coincides with the result of Yang and Norris [5] obtained by another method. Figure 3 illustrates the frequency 
dependence of the normalized scattering cross section $\sigma$ for composite III with an ideally bonded quasi-square inclusion for different values of the parameter $\gamma$ determining the rounding according to (19). For the rounding parameter $\gamma=0.175$ the result is compared with that given by Shindo et al. [24]. A perfect agreement is obtained in this case.

The effective dynamic properties of composites in the zero-frequency limit should approach the corresponding static ones. Consider a composite with randomly distributed and ideally bonded circular fibers, where analytical solutions in the static case are available. In particular, the following relations for the static effective material parameters $\rho, \lambda$ and $\mu$ are taken from [25]

$$
\begin{gathered}
\rho=\rho_{1}(1-\varepsilon)+\rho_{2} \varepsilon, \quad \mu=\mu_{1}+\varepsilon \frac{\mu_{1}\left(\mu_{2}-\mu_{1}\right)}{\mu_{1}+(1+\varepsilon) b\left(\mu_{2}-\mu_{1}\right)}, \\
\lambda=\lambda_{1}+\mu_{1}+\varepsilon \frac{\left(\lambda_{1}+2 \mu_{1}\right)\left(\lambda_{2}-\lambda_{1}+\mu_{2}-\mu_{1}\right)}{\lambda_{1}+2 \mu_{1}+(1-\varepsilon)\left(\lambda_{2}-\lambda_{1}+\mu_{2}-\mu_{1}\right)}-\mu, \quad b=\frac{\lambda_{1}+3 \mu_{1}}{2\left(\lambda_{1}+2 \mu_{1}\right)} .
\end{gathered}
$$

From equations (20) the static counterpart of the average phase velocity can be obtained as $c_{p}^{s t}=[(\lambda+2 \mu) / \rho]^{1 / 2}$. The differences between the values of $\bar{c}$ from equation (18) for $k_{p 1} a=0.05$ and the quantity $c_{p}^{s t} / c_{p 1}$ calculated by equations (20) for the epoxy/glass composite (composite I) with circular fibers of a volume fraction $\varepsilon \leq 0.15$ are always less than $0.5 \%$.

Figures 4-6 depict the frequency dependence of the normalized attenuation coefficient $\bar{\alpha}$ and the normalized effective velocity $\bar{c}$ of plane P-waves in an epoxy/glass composite (composite I) with randomly distributed fibers of circular (Figure 4, $\gamma=0$ ), elliptical (Figure 5, $N=1, \gamma=0.25$, the ratio of the axes is 0.6 ) and square (Figure 6, $N=3, \gamma=1 / 9$ ) shapes and different sizes of the debonding. The volume fraction of the distributed inclusions in the matrix is $\varepsilon=0.1$. The global frequency dependence of the effective characteristics shows a similar tendency for different shapes of the debonded fibers. In the low frequency range the attenuation coefficient $\bar{\alpha}$ increases and the normalized effective velocity $\bar{c}$ decreases when the crack size increases. We observe also a sharp increase of the attenuation coefficient $\bar{\alpha}$ when the frequency increases. As expected [6], the behavior of the effective characteristics of the composite shows a resonance property at a very low frequency when the neck of the bonded interface becomes sufficiently small. In the medium frequency region $\left(2<k_{p 1}<4.5\right)$ the attenuation coefficient $\bar{\alpha}$ decreases when the debonding size increases.

Figures 7 and 8 illustrate the frequency dependence of the normalized effective velocity $\bar{c}$ of Pwaves in composite I with randomly distributed fibers of square shape for different volume fraction $\varepsilon$ 
of the fibers. The perfect or ideal contact of the composite components (Figure 7) and the case of debonding on one side of a square inclusion (Figure 8) are considered. Note here that the normalized attenuation coefficient $\bar{\alpha}$ practically does not depend on the parameter $\varepsilon$. The curves show a rather complex behavior, which can be identified by the quite tangled effects of the fibers stiffness on the average phase velocity at different frequencies. It should be mentioned here that similar phenomena for layered elastic structures, including the behavior of group wave velocities, were investigated in details by Zakharenko [26-28]. In the low frequency range (say $k_{p 1} a<0.9$ ) and when increasing the parameter $\varepsilon$, the effective velocity increases for the ideal contact case and furthermore $\bar{c}>1$, while it decreases for the case with debonded fibers and in addition $\bar{c}<1$ in this case. In the medium frequency region $\left(1<k_{p 1} a<5\right)$ the dependence of the effective velocity on the volume fraction is alike for cracked and uncracked composites. Moreover, we observe a "neutral" frequency or normalized wavenumber $k_{p 1} a \approx 3$, at which the volume fraction does not influence the value of the effective velocity $\bar{c}$ both for ideal and imperfect contact. Here and hereafter $\bar{c} \rightarrow 1$ as the normalized wavenumber tends to $k_{p 1} a \rightarrow \infty$, which means that the wave velocity of plane P-waves in the shortwave limit coincides with their velocity in the matrix.

The normalized attenuation coefficient $\bar{\alpha}$ and the normalized effective velocity $\bar{c}$ versus the dimensionless wavenumber $k_{p 1} a$ are presented in Figures 9-11 for composite I with aligned fibers of the above-mentioned circular (Figure 9), elliptical (Figure 10) and square (Figure 11) shape and different angles of P-wave incidence. We assume that in all considered cases there is either an ideal contact (dotted curves) between the composite components or a debonding with the angles $\theta_{1}=-\theta_{0}=45^{\circ}$ (solid curves). The volume fraction of the distributed inclusions in the matrix is taken as $\varepsilon=0.1$. Only two angles of $\mathrm{P}$-wave incidence $\theta_{i n}=0^{\circ}$ and $\theta_{i n}=90^{\circ}$ along the principal axes of fibers are considered. For aligned fibers the dependence of the effective characteristics on the direction of wave incidence manifests a macroscopic anisotropy of the considered composites with the exception for the composite with ideally bonded circular fibers. This situation is not included in Foldy's theory which does not take the coupling of P- and S-waves and changes in polarization into account. However, for a wave travelling in a symmetry direction there is no change in polarization in the forward scattering and Foldy's theory can be expected to hold [23]. This is the situation for $\theta_{\text {in }}=0^{\circ}$, but for $\theta_{i n}=90^{\circ}$ the results have to be regarded as a further approximation. In the low frequency range the largest value of $\bar{\alpha}$ and the smallest of $\bar{c}$ correspond to normal wave incidence with $\theta_{\text {in }}=0^{\circ}$. For 
the higher wave numbers these maximum $\bar{\alpha}$ and minimum $\bar{c}$ values can be reached under oblique wave incidence. A comparison of the results given in Figures 9-11 with the results given in Figures 4-6 shows that in the low frequency range the values of $\bar{\alpha}$ for randomly oriented debonded inclusions are larger than that for aligned debonded inclusions for $\theta_{\text {in }}=90^{\circ}$ but smaller than that for aligned debonded inclusions for $\theta_{i n}=0^{\circ}$. In contrast, the values of $\bar{c}$ for randomly oriented debonded inclusions are smaller than that for aligned debonded inclusions for $\theta_{i n}=90^{\circ}$ but larger than that for aligned debonded inclusions for $\theta_{i n}=0^{\circ}$. At higher frequencies the opposite tendencies for the considered inclusion arrangements are observed in the most cases. An exception is for the debonded elliptical inclusions and $k_{p 1} a>1.7$, where the normalized effective wave velocity $\bar{c}$ for the random inclusion arrangement could be both larger and smaller than the effective wave velocity for the aligned inclusion arrangement depending on the frequency.

\section{Conclusions}

The solution of wave scattering problem by a partially debonded elastic fiber and Foldy's theory for wave propagation in multiphase materials with distributed fibers of dilute concentration are used to predict the effective phase velocities and the attenuation coefficients of plane longitudinal P-wave in 2-

D fiber-reinforced composites. Wave scattering by a single inclusion is investigated by a modified null field approach, taking into account the asymptotic behavior of the solution at the interface crack-tips. Perfectly bonded fibers with circular, elliptical and square cross-sections and debonded fibers with interfacial cracks are considered in the present analysis. To investigate the influences of the wave frequency, the fiber shape, the fiber size, the fiber orientation or the direction of the wave incidence, and the size of interfacial cracks on the effective dynamic parameters, several numerical examples for plane longitudinal elastic wave propagation in infinite elastic composite materials with both randomly oriented and aligned debonded fibers are presented and discussed.

\section{Acknowledgements}

This work was supported by the INTAS (Project No. 05-1000008-7979), which is gratefully acknowledged. The authors wish to express their sincere thanks to the reviewers for their constructive comments. 


\section{References}

[1] J.D. Achenbach, Quantitative Non-Destructive Evaluation, Int. J. Solids Struct. 37 (2000), pp. 13-27.

[2] I. Bond, M. Hucker, P. Weaver, and S. Haq, Mechanical behaviour of circular and triangular glass fibers and their composites, Compos. Sci. Technol. 62 (2002), pp. 1051-1061.

[3] Ch. Zhang and D. Gross, On Wave Propagation in Elastic Solids with Cracks, Computational Mechanics Publications, Southampton, 1998.

[4] H. Sato and Y. Shindo, Influence of microstructure on scattering of plane elastic waves by a distribution of partially debonded elliptical inclusions, Mech. Materials 34 (2002), pp. 401-409.

[5] Y. Yang and A.N. Norris, Shear wave scattering from a debonded fiber, J. Mech. Phys. Solids 39 (1991), pp. 273-294.

[6] Y. Yang and A.N. Norris, Longitudinal wave scattering from a partially bonded fiber, Wave Motion 15 (1992), pp. 43 59.

[7] Y.-S. Wang and D. Wang, Scattering of elastic waves by a rigid cylindrical inclusion partially debonded from its surrounding matrix. II. P and SV cases, Int. J. Solids Struct. 33 (1996), pp. 2817-2840.

[8] Y.I. Kunets, V.V. Matus, V.V. Mykhas'kiv, A. Boström, and Ch. Zhang, Scattering of a SH-wave by an elastic fiber of nonclassical cross section with an interface crack, Mech. Composite Materials 44 (2008), pp. 165-172.

[9] P.C. Waterman, New formulation of acoustic scattering, J. Acoust. Soc. Amer. 45 (1968), pp. 1417-1429.

[10] V. Varatharajulu and Y.H. Pao, Scattering matrix of elastic wave, J. Acoust. Soc. Amer. 60 (1976), pp. 1361-1371.

[11] P. Olsson and A. Boström, Elastic waves propagation in the presence of crack and thin interface layers, J. Tech. Phys. 31 (1990) 393-400.

[12] A. Boström, P. Olsson, and S.K. Datta, Effective plane wave propagation trough a medium with spheroidal inclusions surrounded by thin interface layers, Mech. Materials 14 (1992), pp. 59-66.

[13] V.F. Emets, Ya.I. Kunets, and V.V. Matus, Scattering of SH waves by an elastic thin-walled rigidly supported inclusion, Arch. Appl. Mech. 73 (2004), pp. 769-780.

[14] P.A. Martin, On connections between boundary integral equations and T-matrix methods, Eng. Anal. Bound. Elem. 27 (2003), pp. 771-777.

[15] D.E. Beskos, Boundary element methods in dynamic analysis: Part II (1986-1996), Appl. Mech. Rev. 50 (1997), pp. 149-197.

[16] A. Boström, Review of hypersingular integral equation method for crack scattering and application to modeling of ultrasonic nondestructive evaluation, Appl. Mech. Rev. 56 (2003), pp. 383-405.

[17] A. Boström and P. Olsson, Scattering of elastic waves by non-planar cracks, Wave Motion 9 (1987), pp. 61-76.

[18] L.L. Foldy, Multiple scattering theory of waves, Phys. Rev. 67 (1945), pp. 107-119.

[19] J.D. Achenbach, Wave Propagation in Elastic Solids, North-Holland, Amsterdam, 1973.

[20] M. Abramowitz and I. A. Stegun, Handbook of Mathematical Functions, Dover Publications, New York, 1972.

[21] J.P. Dempsey and G.B. Sinclair, On the singular behavior at the vertex of a bi-materials wedge, J. Elast. 11 (1981), pp. 317-327.

[22] V.A. Kondrat'ev and O.A. Oleinik, Boundary-value problems for partial differential equations in non-smooth domains, Russ. Math. Surv. 38 (1983), pp. 1-86.

[23] A.S. Eriksson, A. Boström, and S.K. Datta, Ultrasonic wave propagation through a cracked solid, Wave Motion 22 (1995), pp. 297-310. 
[24] Y. Shindo, T. Nakamura, and F. Narita, The application of the boundary element method to the problem of wave diffraction from a diamond shaped inclusion, Open Mech. J. 2 (2008), pp. 62-66.

[25] Z. Hashin and R.W. Rosen, The elastic moduli of fiber-reinforced materials, J. Appl. Mech. 31 (1964), pp. $223-232$.

[26] A.A. Zakharenko, Dispersive Rayleigh type waves in layered systems consisting of piezoelectric crystals bismuth silicate and bismuth germanate, Acta Acustica united with Acustica 91 (2005), pp 708 - 715.

[27] A.A. Zakharenko, Analytical studying the group velocity of three-partial Love (type) waves in both isotropic and anisotropic media, Non-destructive Testing and Evaluation 20 (2005), pp. 237 - 254.

[28] A.A. Zakharenko, On cubic crystal anisotropy for waves with Rayleigh-wave polarization, Non-destructive Testing and Evaluation 21 (2006), pp. $61-77$.

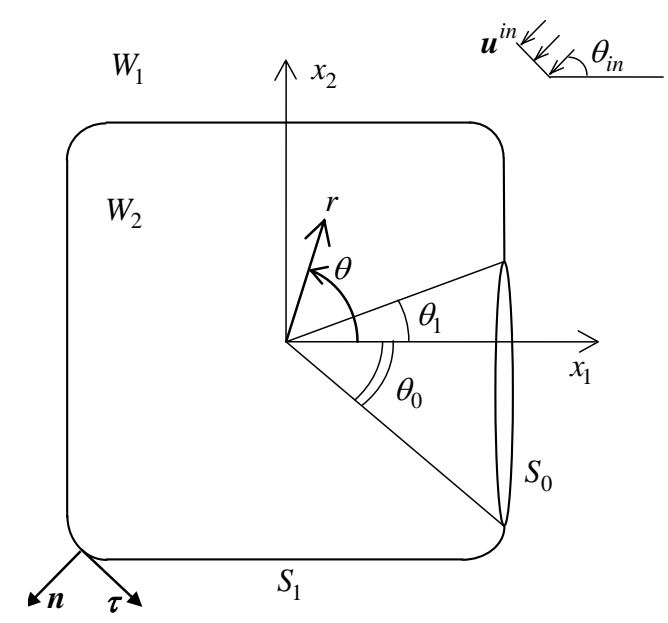

Fig.1. Geometry of the scattering problem. 


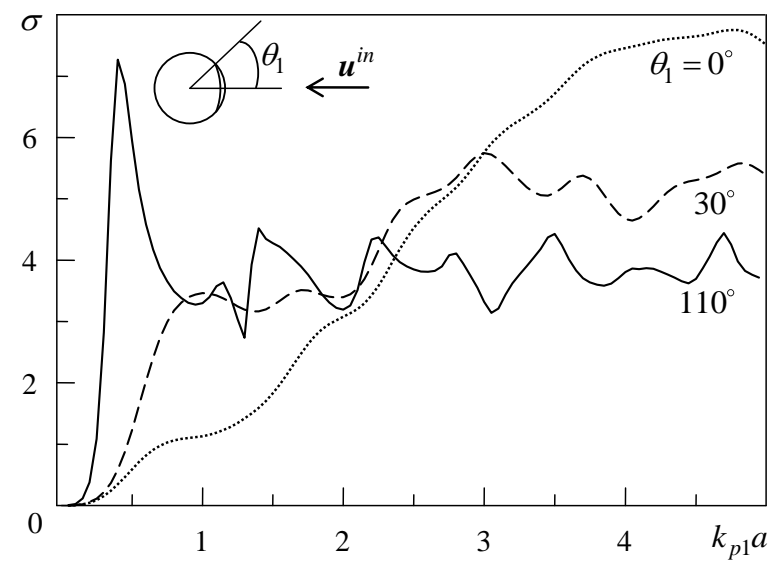

Fig. 2. The scattering cross-section for a partially debonded circular fiber in an epoxy/glass composite (composite II) with $\theta_{i n}=0^{\circ}$ and $\theta_{1}=-\theta_{0}$.

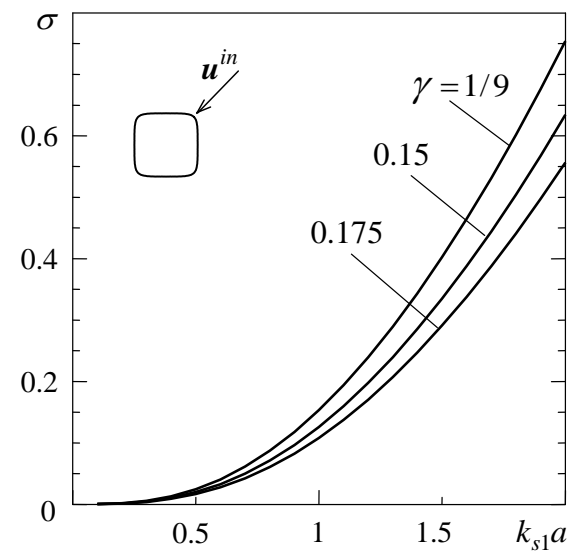

Fig. 3. The scattering cross-section for an ideally bonded square fiber in an $\mathrm{Al} / \mathrm{SiC}$ composite (composite III) with $\theta_{\text {in }}=45^{\circ}$. 

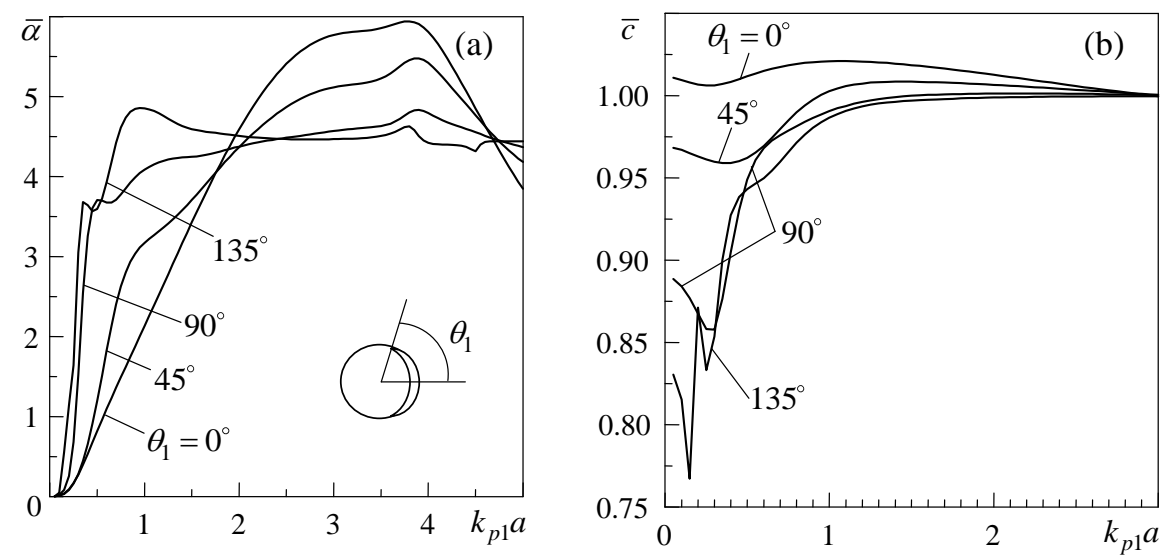

Fig. 4. Random distribution of debonded cylindrical fibers with $\theta_{0}=-\theta_{1}$ in an epoxy/glass composite (composite I). Effects of the crack size on the wave attenuation (a) and phase velocity (b) versus the dimensionless wavenumber.
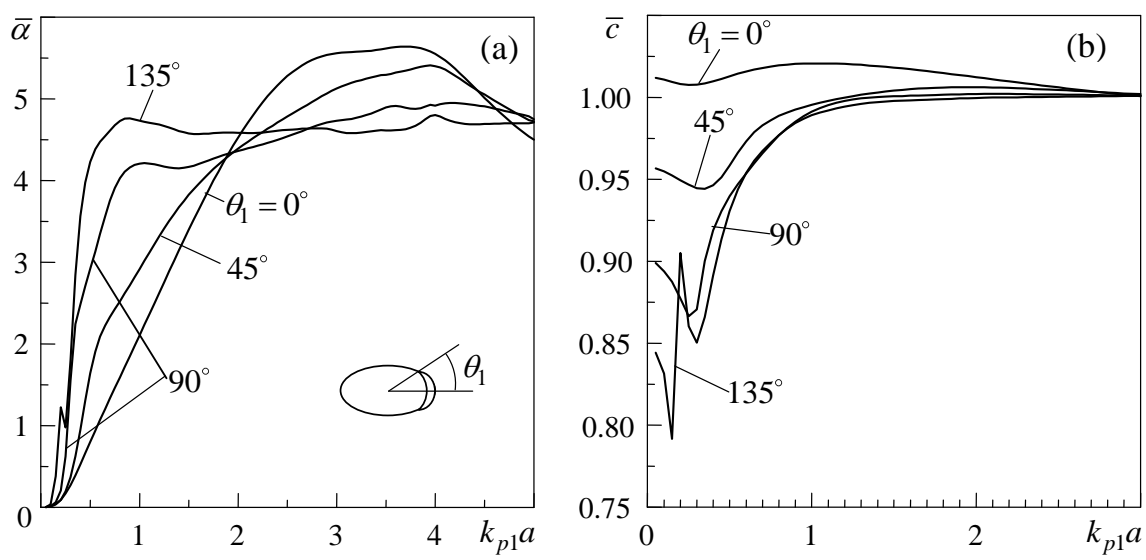

Fig. 5. Random distribution of debonded elliptical fibers with $\theta_{0}=-\theta_{1}$ in an epoxy/glass composite (composite I). Effects of the crack size on the wave attenuation (a) and phase velocity (b) versus the dimensionless wavenumber. 

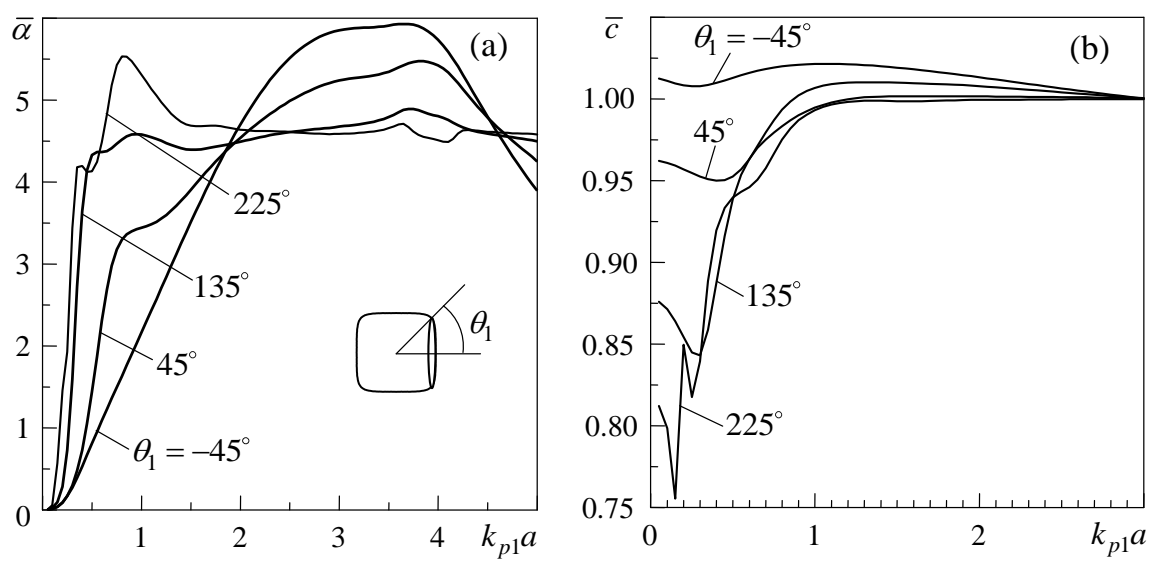

Fig. 6. Random distribution of debonded square fibers with $\theta_{0}=-45^{\circ}$ in an epoxy/glass composite (composite I). Effects of the crack size on the wave attenuation (a) and phase velocity (b) versus the dimensionless wavenumber.

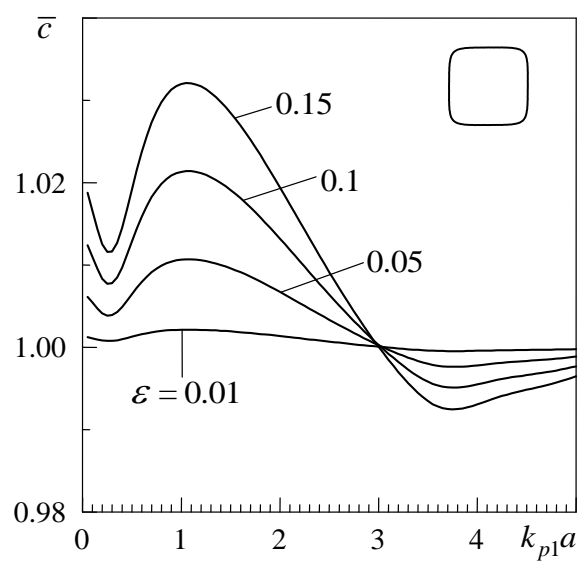

Fig. 7. Random distribution of ideally bonded square fibers in an epoxy/glass composite (composite I).

Effect of volume fraction $\varepsilon$ on phase velocity versus the dimensionless wavenumber. 


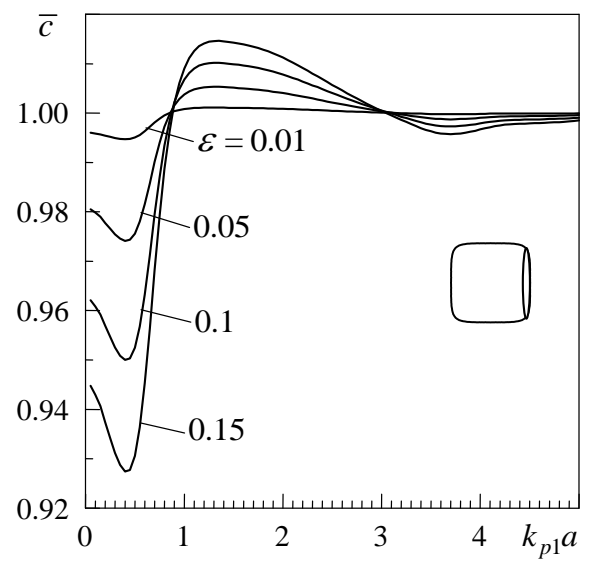

Fig. 8. Random distribution of partially debonded square fibers with $\theta_{1}=-\theta_{0}=45^{\circ}$ in an epoxy/glass composite (composite I). Effect of volume fraction $\varepsilon$ on phase velocity versus the dimensionless wavenumber.
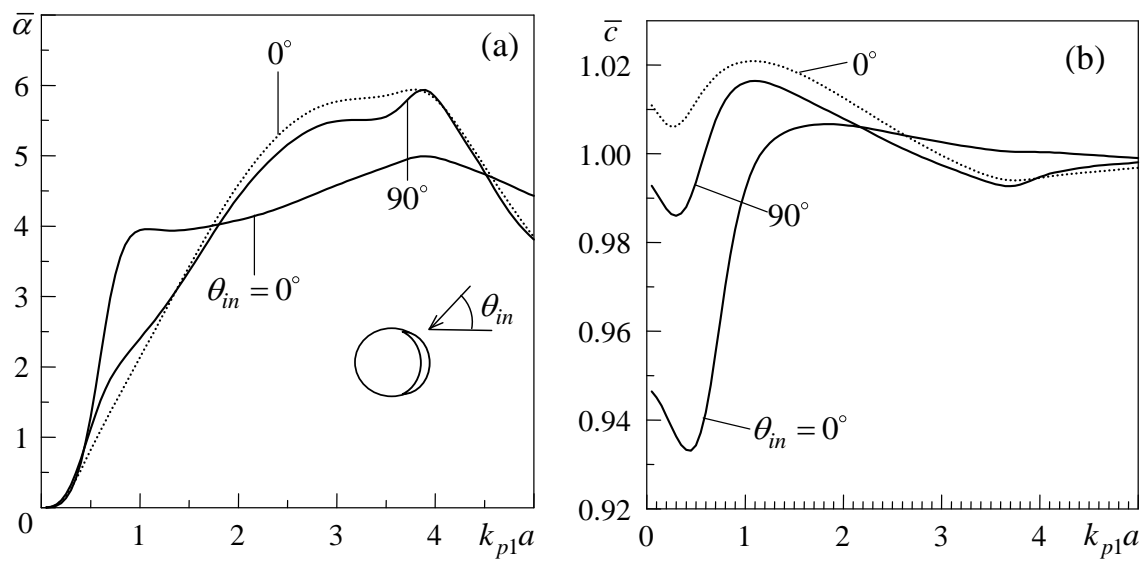

Fig. 9. Aligned circular fibers with (solid curves) and without (dotted curves) debonding in an epoxy/glass composite (composite I). Effects of the incidence direction on the wave attenuation (a) and phase velocity (b) versus the dimensionless wavenumber for $\theta_{1}=-\theta_{0}=45^{\circ}$. 

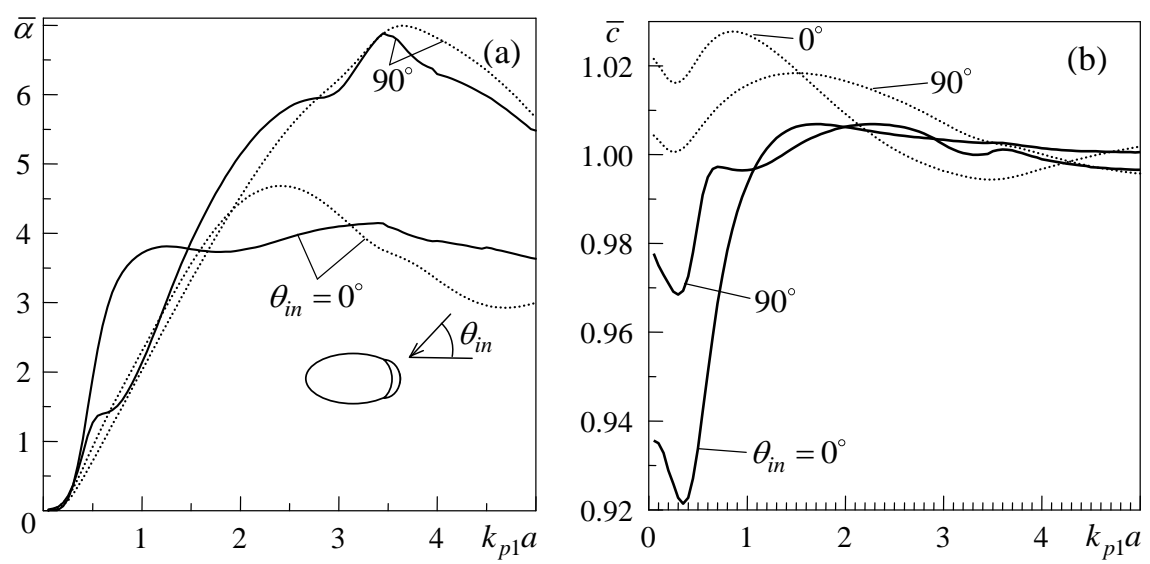

Fig. 10. Aligned elliptical fibers with (solid curves) and without (dotted curves) debonding in an epoxy/glass composite (composite I). Effects of the incidence direction on the wave attenuation (a) and phase velocity (b) versus the dimensionless wavenumber for $\theta_{1}=-\theta_{0}=45^{\circ}$.
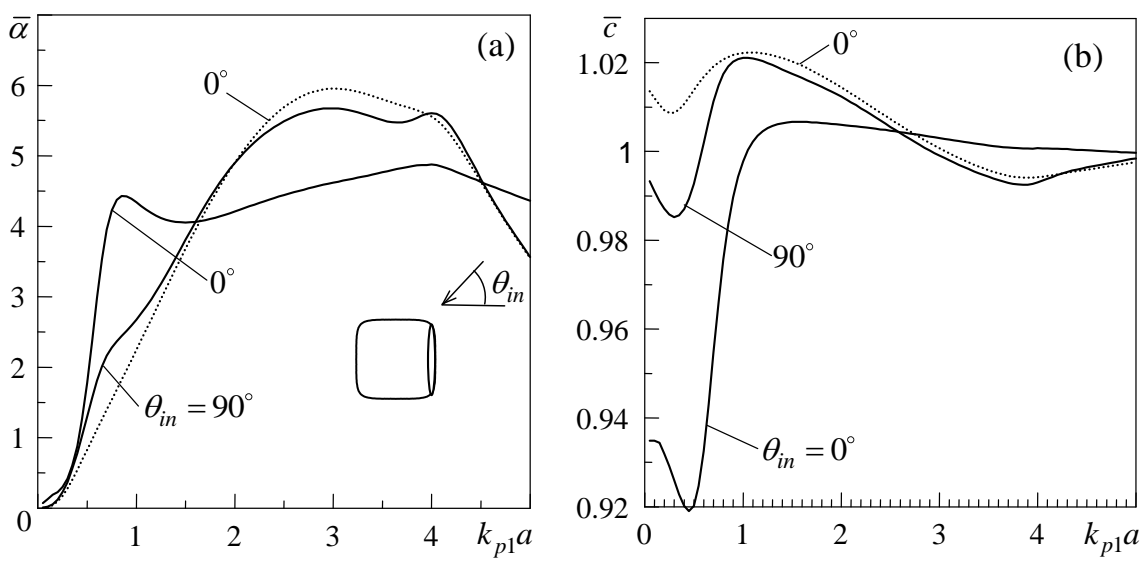

Fig. 11. Aligned square fibers with (solid curves) and without (dotted curves) debonding in an epoxy/glass composite (composite I). Effects of the incidence direction on the wave attenuation (a) and phase velocity (b) versus the dimensionless wavenumber for $\theta_{1}=-\theta_{0}=45^{\circ}$. 